\title{
R-Loops in Motor Neuron Diseases
}

\author{
Martina G. L. Perego ${ }^{1} \&$ Michela Taiana ${ }^{1} \&$ Nereo Bresolin ${ }^{1} \&$ Giacomo P. Comi ${ }^{1} \&$ Stefania Corti $^{1}$
}

\begin{abstract}
$\mathrm{R}$ loops are transient three-stranded nucleic acid structures that form physiologically during transcription when a nascent RNA transcript hybridizes with the DNA template strand, leaving a single strand of displaced nontemplate DNA. However, aberrant persistence of R-loops can cause DNA damage by inducing genomic instability. Indeed, evidence has emerged that R-loops might represent a key element in the pathogenesis of human diseases, including cancer, neurodegeneration, and motor neuron disorders. Mutations in genes directly involved in R-loop biology, such as SETX (senataxin), or unstable DNA expansion eliciting R-loop generation, such as C9ORF72 HRE, can cause DNA damage and ultimately result in motor neuron cell death. In this review, we discuss current advancements in this field with a specific focus on motor neuron diseases associated with deregulation of R-loop structures. These mechanisms can represent novel therapeutic targets for these devastating, incurable diseases.
\end{abstract}

Keywords R-loops · DNA damage $\cdot$ Motor neuron disease $\cdot$ Amyotrophic lateral sclerosis $\cdot$ Spinal muscular atrophy

\section{Introduction}

The etiopathogenesis of neurodegenerative diseases, such as Alzheimer's disease, Parkinson's disease, and amyotrophic lateral sclerosis (ALS), remains largely unknown, which hampers the rational development of effective treatments. These disorders have in common the selective loss of a specific neuronal subpopulation. In several neurodegenerative diseases, this progressive cell death has been related to a common neuropathological feature, namely the accumulation of proteins that aggregate in cells due to their misfolded or altered structure $[1,2]$. Although a common underlying pathological feature of many neurodegenerative diseases is the accumulation of protein aggregates, it is not clear whether such structures are universally toxic to neurons. Indeed, recent studies have highlighted the possible role of RNA alterations in the pathogenesis of these disorders [3]. In particular, the defect in the resolution of transcriptional R-loops, a consequence of abnormal mRNA metabolism, is thought to be responsible for DNA damage and genome instability [4-6].

* Stefania Corti

stefania.corti@unimi.it

${ }^{1}$ Dino Ferrari Centre, Neuroscience Section, Department of Pathophysiology and Transplantation (DEPT), Neurology Unit, IRCCS Foundation Ca' Granda Ospedale Maggiore Policlinico, University of Milan, Milan, Italy
This notion represents a new perspective in the comprehension of the pathogenesis of these diseases and may possibly open paths to new therapeutic targets.

R-loops are transient nucleic acid structures composed of three strands (mRNA, template DNA strand, and not-template DNA strand) that form during the transcriptional process. In the course of transcription, dsDNA is unwound, and one strand of the DNA double helix is copied into a complementary RNA transcript. Within the transcription bubble, the two strands of DNA are physically separated, and a transient 8-bp RNA/DNA hybrid forms [7]. In most cases, the two DNA strands reanneal as the RNA is synthesized along the DNA. However, R-loops can develop in particular over DNA regions characterized by high $\mathrm{G}$ density, negative supercoiling, and DNA nicks [8] during transcription. The exact mechanism of R-loops generation remains unclear. In specific circumstances, the RNA:DNA hybrid could be maintained and extended as the polymerase moves forward, leaving the other DNA strand unbound. Another mechanism proposed for Rloops generation is the thread-back model. In this model, the nascent RNA transcript exiting the active site of RNA polymerase can anneal to the template DNA strand before the reannealing of the two strands of the DNA duplex, generating a RNA:DNA hybrid that is more stable than dsDNAs [9] and discarding the non-template, single-stranded DNA [10-12].

A number of cellular processes are controlled by the programmed formation of R-loops, e.g., Ig class switch recombination and termination of transcription [12]. Normally, R-loop 
levels are under tight control, and R-loop formation is limited by the binding of RNA-binding proteins to the forming RNA, reducing its hybridization to the DNA template. In addition, R-loops can be actively resolved by the action of RNA/DNA helicases and RNase H1 [13, 14].

Defects in the mechanisms responsible for the resolution of R-loops or the presence of a G-rich sequence in the nontemplate DNA that stabilizes R-loops by the formation of G-quadruplexes can lead to aberrant and potentially harmful R-loops; impairment of R-loop levels may induce alteration of chromatin structure and transcriptional modification, DNA double-strand breaks, and consequent cellular damage $[13,15,16]$. Indeed, knockdown of RNA splicing and transport factors is associated with increased R-loop formation and consequential DNA damage $[17,18]$.

In recent years, an increasing number of studies $[12,19]$ have highlighted the altered metabolism of R-loops as a possible source of genome instability involved in the pathogenesis of a variety of diseases, including cancers and neurodegenerative disorders [20].

Currently, the following main mechanisms have been acknowledged to be responsible for nonphysiological R-loop formation in neurodegenerative disorders, depending on the genetic defect characterizing the diseases (Table 1): (1) mutations in genes encoding factors playing a role in the R-loop generation and resolution pathways, i.e., senataxin, a RNA/ DNA helicase responsible for R-loop resolution at transcription termination sites [22, 32] and (2) the presence of pathogenic repeat expansion (Fig. 1). Numerous repeat sequences causing neurodegenerative diseases form stable R-loops that subsequently contribute to repeat instability [33-36]. For instance, the CAG/CTG expansion that causes greater than 13 different diseases, including Huntington's disease (HD), myotonic dystrophy type 1 (DM1), and multiple spinocerebellar ataxias (SCAs), creates R-loops and promotes genetic instability [34].

Furthermore, the expansion of the GAA repeats in the first FXN gene that causes Friedreich ataxia is thought to be capable of promoting R-loop formation and stabilization given its G-rich content, resulting in focal DNA breaks [21].
Despite the fact that these mutations occur in ubiquitously expressed genes, these diseases present primarily neurologic deficits and most strikingly motor neuron signs, highlighting a particular sensitivity of the nervous system and motor neurons to defects in R-loop metabolism and genome stability.

In this work, we intend to present and discuss recent advancements in elucidating the role of R-loops in motor neuron disorders and their possible therapeuticimplications.

\section{R-Loops in Motor Neuron Disease}

\section{Amyotrophic Lateral Sclerosis}

Amyotrophic lateral sclerosis (ALS) is a neurodegenerative disease characterized by an adult onset and a fatal outcome typically 3 to 5 years after diagnosis. ALS is characterized by signs and symptoms due to the degeneration of both upper and lower motor neurons located in the brain and in the spinal cord, respectively, that clinically leads to progressive muscle weakness and paralysis until the end stage with death from respiratory failure [37].

To date, riluzole and edavarone are the only two approved therapies for ALS. However, these agents only modestly slow disease progression, and all patient management relies on symptomatic therapies [37, 38].

Although mutations in different genes with different neuronal functions cause some ALS cases, most patients exhibit sporadic disease and develop the disease through unknown mechanisms [39]. Recent findings suggest an involvement of R-loops in the pathogenesis of the ALS forms with known genetic causes [20,40]. This association has not been demonstrated in sporadic ALS forms without identified genetic causes to date. Here, we review the possible associations between ALS causative genes and pathological R-loop formation. This information could contribute to understanding the disease pathogenesis, identifying common pathogenic mechanisms for different types of ALSs, and subsequently revealing novel therapeutic targets.
Table 1 Different gene mutations/alterations that lead to R-loop formationinmotorneuron diseases

\begin{tabular}{lll}
\hline & Disease & Mechanism \\
\hline C9orf72 HRE & ALS [21] & R-loop stabilization (G4-DNAs) \\
ATXN2 repeats expansion & ALS [6, 22] & Alteration of R-loop resolution \\
SETX mutations & ALS4 [23], AOA2 [24-27] & Altered RNA/DNA helicasefunction \\
TDP-43/FUS mutations & ALS [22, 28] & Altered RNA binding protein function \\
SMN mutations & SMA [29, 5] & ATNX 2 and SMN sequestration \\
& & Altered splicing, consequential R-loop \\
IGHMBP2 mutations & increase \\
\hline
\end{tabular}


Fig. 1 Role of R-loops in motor neuron disease pathogenesis. Rloops are transient three-stranded nucleic acid structures composed of a nascent RNA transcript hybridized with the DNA template strand and a single strand of displaced nontemplate DNA. The figure illustrates the physiological and dysfunctional consequences of their formation

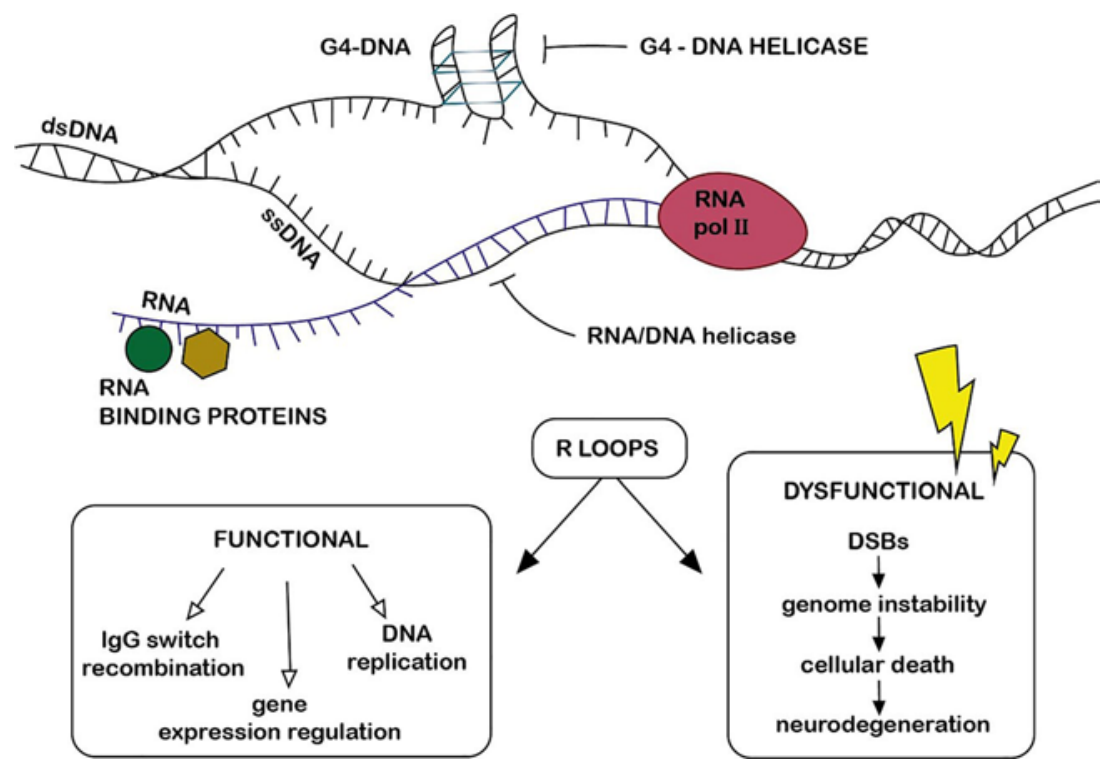

\section{C9ORF72}

Among all of the genes causing ALS, a hexanucleotide repeat expansion (HRE) GGGGCC in the first intron of the C9ORF72 (C9) gene is the most common genetic cause of both familial and sporadic ALS and frontotemporal dementia (FTD) [41-43]. Less than 30 hexanucleotide repeats are generally found in normal subjects, whereas up to thousands are detected in ALS patients [44].

The pathogenic mechanisms leading to the disease linked to $C 9$ repeats expansion and how R-loop generation at the locus may be implicated are mostly unknown. However, different mechanisms have been proposed. G-rich expansions in C9's first intron can form G-quadruplexes in the displaced DNA strand (G4-DNA), which stabilizes the R-loops that form preferentially at $G$-rich sites. Additionally, $C 9$ transcripts tend to form RNA G-quadruplexes (G4-RNA). Moreover, these G4-DNA sequences interfere with RNA polymerase II activity, causing premature interruption of transcription associated with the formation of truncated mRNAs [35, 45-48]. Haeusler et al. (2014) observed that R-loop resolution after RNase $\mathrm{H}$ treatment reduces the accumulation of abortive transcripts, suggesting that both of the secondary structures originating from C9 HRE (G4-DNA and R-loops) contribute to the impairment of the transcriptional process. Afterwards, the truncated G4-transcripts tend to aggregate in the cellular nucleus and cytoplasm and bind to proteins. In particular, sequestration of the ribonucleoprotein nucleolin leads to its mislocalization as observed in motor neurons differentiated from C9 HRE ALS-induced pluripotent stem cells (iPSC), and nucleolar stress is detectable in the cerebral cortex tissue of affected patients [45].

There are many G/C combinations in addition to G4C2 HRE of $C 9$ that are responsible for R-loop formation and G4 structures. To date, G4C2 repeat expansion has not been identified in other genes known to cause ALS or FTD [49]. However, this does not mean that similar G/C combinations causing R-loop formation and G4 structures would not be identified in the $\sim 50 \%$ of ALS cases for which we do not have a genetic explanation to date. On the other hand, $C 9$ HRE is associated with other neurological disorders, such as Alzheimer disease [43] and Huntington disease [50], suggesting a possible role of R-loops and G4 structures in these disorders.

Although C9 HRE could explain the increase in R-loops in ALS patients' cells, the role of R-loops in ALS pathogenesis is largely undetermined. A possible mechanism is related to the impairment of the transcription of the $C 9$ gene, and this impairment may be related to R-loop formation. C9 expression is reduced in mutated C9 HRE cells, where the presence of repressive chromatin and hypermethylated regions on the $C 9$ gene has been observed [51-56]. Both of these events can cause reduced $C 9$ expression. Multiple studies have suggested that also R-loops can negatively regulate transcription in vitro and in vivo through different mechanisms, including RNA polymerase pausing, antisense transcription, and histone methylation [57]. R-loop formation is also observed in unmethylated human CpG island promoters [58].

Transcription of $C 9$ expansions is typically bidirectional, and the number of R-loops is increased in genes producing antisense RNA. Thus, the inhibition of $C 9$ transcription could be explained by the bidirectional transcription/chromatin modification due to the presence of numerous R-loops [59]. However, the exact mechanism needs to be further investigated. Recent data suggest that R-loops may not be necessary for the DNA hypermethylation linked with C9 HRE. In fact, preventing R-loop formation in neurons did not halt heterochromatinization of the HRE [60]. 
Walker and colleagues (2017) identified genomic instability driven by persistent accumulation of R-loops as a pathological mechanism of C9 HRE ALS [61]. They observed that overexpression of $C 9$ expansions in human fibroblasts led to increases in R-loops and consequent DNA double-stranded breakages (DSBs). Insertion of 102 C9 repeats resulted in the formation of RNA foci but not dipeptide repeats (DPRs). This work describes an increase in R-loop formation induced by DPR expression, even though the mechanisms of this event are not known. However, they observed that R-loops were also formed in cells transfected with constructs that did result in the translation of DPRs 34 and 69, suggesting that different mechanisms mediate the formation of R-loops [61]. Remarkably, overexpression of senataxin, an RNA helicase that resolves R-loops, reduced the number of breakages and even inhibited DNA damage-mediated cell death. Together, these data support the notion that HRE leads to R-loop accumulation, which causes DNA damage and consequential cell death. The authors also identified a defect in the DNA repair capacity of the cells expressing C9 HRE due to defective signaling of the ataxia telangiectasia (ATM) protein, one of the principal DNA repair kinases, highlighting the relevance of genome instability in C9 pathogenesis [61]. Interestingly, the researchers also demonstrated R-loop accumulation, double-stranded breaks presence, and weak ATM activation in neurons from mice treated with viral vectors encoding for the repeat expansions and in postmortem spinal cord tissue from ALS patients. Finally, this study supports the notion that targeted modulation of R-loop homeostasis by R-loopspecific helicases, such as senataxin, or DSB repair kinetics by chromatin-modulating drugs may represent new therapeutic strategies for $C 9$-associated diseases [61].

Overall, despite the possible illustrated explanations about $\mathrm{R}$-loops and $C 9$ connection, further research is still needed to clarify these topics.

\section{Ataxin-2}

The polyglutamine (polyQ) diseases are a group of neurodegenerative disorders caused by expansion of the trinucleotide repeat cytosine-adenine-guanine (CAG), encoding a long polyQ tract in specific proteins. In particular, polyQ expansions in the ataxin-2 (ATXN-2) gene are associated with two different neurological diseases, ALS [11, 62] and spinocerebellarataxia type 2 (SCA2) [63, 64]. Normally, ATXN2 contains 22 CAG repeats. Although significant expansions of CAG have been reported in SCA2, it has recently been demonstrated that intermediate-length repeats (27-33 repeats) might have a role in the pathogenesis of ALS [11, 62, 65].

Salvi et al. (2014) observed that Pbp1, the ortholog of ATXN2 in yeast, regulates R-loop generation by inhibiting deleterious R-loop formation at G4-DNA sites in the intergenic spacer regions of the ribosomal DNA repeats and at telomeres [66]. Through these actions, Pbp1 maintains ribosomal DNA stability [66]. In humans, ATXN2 mightalso inhibit R-loop formation at some loci although ATXN2 is mainly a cytoplasmic protein [11].

ATXN2 physically interacts with TAR DNA-binding protein 43 (TDP-43) in a RNA-dependent manner, influencing the aggregation propensity of TDP-43. Reduction of ATXN2 levels in TDP-43 ALS mice models results in a decrease in TDP-43 inclusions [67]. The strength of the ATXN2/TDP-43 interaction is proportional to the length of the polyQ expansions [62]; thus, intermediate-length repeats could increase TDP-43 pathological modification. TDP-43 is one of the major disease-associated proteins in ALS and FTD [68]. Wildtype TDP-43 localizes in the nucleus, whereas mutated TDP43 aggregates in the cytoplasm and is associated in neurons with stress granules and cytoplasmic aggregates containing translationally inert RNA and associated proteins that are markers of ALS [68, 69]. Other proteins accumulate in cytoplasmic stress granules of degenerating neurons of ALS patients, such as fused in sarcoma (FUS), a protein that plays an important role in ALS and FTD pathogenesis [65, 70]. Both TDP-43 and FUS bind to DNA and RNA and are associated with numerous nuclear and cytoplasmic steps of mRNA maturation and transport [24, 71]. After stress induction, ATNX2 and TDP-43 both relocate to stress granules $[25,69]$ together with FUS [65]. Intermediate-length polyQ expansions in ATXN2 might be responsible for the sequestration of TDP43 into cytoplasmic aggregates, altering its ability to bind DNA and RNA. Otherwise, mutated TDP-43 and FUS are potentially able to sequester ATXN2 into cytoplasmic stress granules, altering its possible function as a suppressor of Rloop formation [40]. It would be interesting to better understand the supposed role of ATXN2 in R-loop metabolism and to assess R-loop accumulation in SCA2 cells, particularly in those cells directly affected by the disease, such as human iPSCs-derived Purkinje cells. Moreover, studies of the direct function of TDP-43 and FUS in R-loop regulation in the nucleus might clarify the relationship between ATXN2 polyQ expansions and ALS.

\section{Senataxin}

Senataxin (SETX) is an RNA/DNA helicase that is mutated in two different neurological diseases, the recessive disorder ataxia with oculomotor apraxia type 2 (AOA2) [26] and a dominant juvenile form of amyotrophic lateral sclerosis (ALS4) [22] that has suggestive similarities with spinal muscular atrophy (SMA) and is characterized by the death of motor neurons in early childhood [22]. Identification of rare atypical forms of AOA2 and ALS4 that are also associated with SETX mutations enlarge the phenotypic spectrum that correlates with mutations in this gene [27,72]. 
As for ATX2, the yeast homologous gene Sen1 was studied to understand SETX function and pathology. Sen1 has a role in the termination of transcription of noncoding RNAs and some mRNAs [23, 73] and the suppression of R-loop formation and transcription-associated recombination [74]. These findings were confirmed also for SETX, which has a role in the resolution of R-loops typically formed at the transcription termination pause site in the human $\beta$-actin gene [16] and other genes via BRCA1-dependent activity [75]. SEXT/ BRCA1 recruitment to the 3' pause site avoids the accumulation of R-loops that could induce DNA DSBs [75]. Both Sen1 and SETX are responsible for the prevention of genome instability caused by R-loops via an unknown mechanism. This finding was also confirmed by the fact that in response to replication stress, SETX aggregates to form foci and relocalizes to R-loops with the RNA nuclear exosome, a 3' to 5' exoribonuclease complex that has a role in RNA quality control [76, 77]. SETX interaction with the exosome subunit Rrp45 [77] is dependent on SETX sumoylation, which is altered by AOA2 SETX mutations but not by ALS4 SETX mutations [77, 78]. Thus, it is possible that SETX unwinds RNA/ DNA hybrid structures at R-loop sites, whereas the nuclear exosome degrades the released RNAs that might rehybridize with portions of the DNA or exhibits some toxic effects. The reason why this action important in AOA2 pathogenesis and not in ALS4 pathogenesis remains unclear.

Nevertheless, the role of R-loop formation itself in AOA2 pathogenesis remains under investigation. Todate, conflicting results are available in the literature. SETX knock out mice accumulate R-loops; however, in this context, R-loop accumulation does not lead to any phenotypic characteristics of AOA2 and otherwise causes male sterility [79, 80]. In fact, $\mathrm{R}$-loops seem to increase in germ cells but not in neuronal cells in SETX, ATM (the gene causing ataxia teleangiectasia), TDP1 (responsible for spinocerebellar ataxia with axonal neuropathy 1), and APTX (that causes AOA1) knock out mice [80]. However, AOA2 cells exhibit a reduced telomeres length that could be explained by the role of SETX in telomere stability given that R-loops and G4-DNA form at telomeres [28, 29]. Indeed, accumulation of R-loops with non-coding RNA BTERRA $\wedge$ at telomeric regions of DNA activates proteins involved in recombination [81].

Overall, these data could provide some evidence for AOA2 pathogenesis but not for ALS4.

The clinical similarities between ALS4 and SMA and the finding that SMN (the protein responsible for SMA pathogenesis) recruits SETX to transcription termination sites [6] suggest a possible shared pathogenetic pathway involving Rloops between these two disorders. An R-loop pattern in ALS4 SETX-mutated cells was recently investigated by Grunseich and colleagues [82]. This team demonstrated that ALS4 patient cells with the heterozygous L389S SETX mutation have surprisingly less R-loops. The mechanism through which this mutation causes a decrease in the number of Rloops remains unclear. The authors suggest a possible SEXT gain of function connected with the mutation, which is consistent with the dominant pathway of transmission of SETX mutations in ALS4 [82]. Depletion of R-loops at gene promoters due to this SETX mutation affects gene expression. In particular, in ALS4 cells, low levels of R-loops reduce the expression of $B A M B I$, a negative regulator of transforming growth factor $\beta$ (TGF- $\beta$ ) and consequently activate the TGF- $\beta$ pathway through an increase in BAMBI promoter methylation. Genome-wide analyses identified greater than 1200 gene promoters that are regulated in their methylation by R-loops, raising interesting questions regarding the possibility that R-loops could act as promoters of transcription [82]. The authors demonstrated that DNA methyltransferase 1 (DNMT1) binds more avidly to double-stranded DNA than to RNA/DNA hybrids, explaining why genes with R-loops in their promoters are less methylated and consequently more transcribed. Therefore, R-loops could represent a mechanism by which nascent mRNAs promote their own transcription by blocking methylation-mediated gene silencing in a positive feedback loop [82]. Recently, Cohen and colleagues observed that SETX is recruited at DSBs that occur in transcriptionally active loci. Genome-wide mapping reveals that R-loops accumulate on the chromatin next to DSBs apart from small regions near DNA ends where they do not bind; these R-loopfree islands induced by the DSB itself are bound by SETX. SETX is not able to resolve DSBs alone but can facilitate genome stability and integrity by the recruitment of RAD 51 that subsequently reduces the illegitimate rejoining of distant DNA ends and ensures cell vitality after DSBs [83]. These new functions of SETX could potentially play a role in AOA2/ALS4 neuropathies.

Finally, there is evidence for a role of SETX in other human diseases, such as ovarian cancer, where SETX is deleted, suggesting a possible role as a tumor suppressor gene mediated through its R-loop-resolving function [84]. However, this hypothesis has not been confirmed by increased tumor susceptibility in AOA2 patients.

RNA-Binding Proteins: TDP-43 and FUS this is subtitle no main title the stile is like Ataxin 2

The RNA-binding proteins TAR DNA-binding protein 43 (TDP-43) and fused in sarcoma (FUS) are both involved in the pathogenesis of familial and sporadic forms of ALS. These proteins can move between the nucleus and the cytoplasm and have important roles in RNA metabolism, such as transcription and mRNA splicing and transport. Moreover, in cases of cellular stress, these proteins can localize to stress granules, which are dynamic sites of translational repression $[85,86]$. The presence of TDP-43 cytoplasmic ubiquitinated aggregates in the brain and spinal cord is a hallmark of ALS. FUS 
is occasionally found in TDP-43 aggregates or can form aggregates independently when it is mutated. Whether TDP-43 and FUS aggregates and stress granules are the same cytoplasmic structures and the exact composition and function of these aggregates remain unknown [87]. Cellular TDP-43 levels are under strict control. TDP-43 regulates its mRNA levels through a negative feedback loop [88]. Instead, TDP43 mutations at the C-terminal region increases its aggregation capability and inhibits its self-regulation based on a negative feedback mechanism [88].

In addition, FUS sequesters SMN in the cytoplasm, causing alterations in snRNA levels and mRNAs splicing. Some of these alterations are responsible for SMN loss, creating a vicious circle [30]. Moreover, when overexpressed, SMN induces stress granules assembly [31]. As previously explained, ATXN2 interacts with FUS, TDP43, and other RNA-binding proteins and localizes within stress granules [40]. As noted, these protein alterations are various and variably interconnected. One potential model to explain all of these interconnections among different genetic defects is a self-reinforcing loop that gains strength with disease progression in which stress granules or cytoplasmic protein aggregates cause the sequestration of proteins normally located in the nucleus, such as TDP-43, ATXN2, and SMN, altering their nuclear functions, in particular their roles in RNA splicing, processing, and transport as well as R-loop metabolism and resolution. R loop accumulation can subsequently lead to genomic instability and accumulation of mutations that can promote stress granules formation, maintaining the cycle [40]. In support of this model, the implications of FUS and TDP-43 in the prevention of R-loop-associated DNA damage has been recently proposed in ALS models [89].

All of these findings and hypotheses lead to numerous other questions. Overall, we reported that alterations in splicing factors, transport proteins, and molecules implicated in RNA processing caused by their aggregation in cytoplasm or by genetic mutations subsequently promote DNA doublestrand breaks via R-loop accumulation. This findingreveals the genetic instability that causes motor neuron death in both SMA and ALS. However, why selective death of motor neurons occurs given that these mutations are located in ubiquitously expressed genes is a very perplexing and problematic question. The sensitivity of this cell type to genomic instability due to an inefficient repair machinery is a possible hypothesis; however, this hypothesis could be incorrect or could represent only one explanation [90].

\section{Spinal Muscular Atrophy}

Spinal muscular atrophies (SMAs) are a group of hereditary diseasesaffectinglowermotorneuronsthatmanifestclinically with progressive proximal paralysis, muscular atrophy, respiratory distress, and death by the age of 2 years in the most aggressive forms of the disease [91]. Today, the term SMA is used commonly to denote 5q SMA or Bproximal spinal muscular atrophy $\wedge$, the most frequent form of the disease, accounting for approximately $95 \%$ of all SMAs [91]. In fact, 5q SMA is the most common monogenic cause of infant mortality due to mutations or deletions of the survival of motor neuron 1 (SMN1) gene located on chromosome $5 q 13$ and results in the subsequent reduction of SMN protein expression. Its inheritance pattern is autosomal recessive [91].

Recent advances in understanding SMA pathogenesis have laid the foundation for new therapeutic approaches. The first drug approved for all types of SMA was nusinersen, a modified antisense oligonucleotide (ASO) that modulates the splicing of SMN2 mRNA transcripts [92]. Moreover, other strategies have been proposed, including gene therapy using adenoassociated virus (AAV) vectors carrying the $S M N$ gene with positive results obtained in the first clinical trial, modulation of SMN2 transcripts, neuroprotection, and targeting of numerous peripheral targets (such as skeletal muscles) [92].

The second most frequent form of SMA is spinal muscular atrophy with respiratory distress (SMARD1), a rare and fatal disease that typically affects children during the first few months of life. SMARD1 is caused by autosomal recessive inherited mutations in the IGHMBP2 gene, which encodes the immunoglobulin $\mu$-binding protein 2 . Mutations in this gene leads to motor neuron degeneration that primarilymanifests with muscular atrophy and diaphragmatic palsy, requiring prompt and permanent ventilation; without ventilation, the disease is fatal in few years [93, 94]. Currently, no approved treatment is available to date, but different therapeutic strategies, such as gene therapy or stem cell transplantation, have exhibited positive results in nmd mice [93, 94].

\section{SMA $5 q$}

SMN is a ubiquitously expressed protein, and its reduction causes selective motor neuron death in SMA. Its functions are numerous, but it seems to exert a critical role in RNA splicing processes [91].

R-loop dynamics can be regulated by splicing factors [12]. Indeed, impaired mRNA splicing driven by loss of spliceosomal components promotes R-loop accumulation and DNA DSBs in neurons in a zebrafish model. Resolution of R-loops after RNase H1 overexpression rescued DNA damage and neuronal apoptosis in zebrafish splicing factor mutants [95]. The widespread splicing alterations that are induced by reductions in SMN protein levels in SMA are potentially associated with impaired formation of transcriptional R-loops and subsequent global DNA damage and stress responses, which could ultimately compromise motor neurons survival.

In splicing pathways, SMN plays a key role in the assembly of the spliceosomal small nuclear ribonucleoproteins 
(snRNPs) that are necessary for intron removal during premRNA splicing [96, 97]. Jangi et al. (2017) observed that human SMN1-silenced SH-SY5Y cells accumulate R-loops and DNA DSBs in vitro mainly over retained introns [90]. Intron retention is defined by the presence of a transcriptconfirmed intron within a transcript-confirmed exon. Interestingly, retained introns are not though to alter transcription but only generate cellular stress. Indeed, in an SMA mouse model, SMN-dependent retained introns did not significantly affect the expression of the genes in which they were located, suggesting that these introns are ultimately removed in the nucleus, probably more slowly than that noted in normal cells. This notion could explain why retained introns do not affect translation but could be sufficient to trigger cellular stress. These observations highlight the greater importance of the process of stochastic intron retention than the specific identity of retained introns [90].

Pervasive intron retention was also observed in SMNdepleted human SH-SY5Y neuroblastoma cells and SMAinduced pluripotent stem cell (iPSC)-derived motor neurons in association with p53 pathway activation and increases in $\mathrm{Y}$ H2A.X and other markers of DNA damage response [90]. The mechanism connecting the reduction in SMN and DNA instability remains unclear. The authors proposed a role for Rloops. In fact, they demonstrated that introns frequently retained after SMN depletion in SH-SY5Y cells were enriched in R compared with spliced introns. They also demonstrated that these retained introns were rich in GC nucleotides that represent valid substrates for R-loop formation [90]. Moreover, resolution of R-loops through RNase H1 overexpression rescued DSBs in SH-SY5Y cells depleted of SMN. Low SMN levels subsequently induce defects in intron removal and R-loop formation, causing genome instability. The mechanism by which this action leads to specific motor neurons death remains undefined. However, the extent of intron retention and R-loop accumulation is probably dependent on SMN expression levels, and this information could be important in intermediate models of SMA (SMA type II and SMA type III) [90].

SMN may also have an active role in R-loop resolution through the recruitment of senataxin [6]. RNA polymerase II, senataxin, and SMN are involved in a pathway for R-loop resolution that could possibly have a role in the pathogenesis of neurodegenerative disorders when altered.

\section{Spinal Muscular Atrophy with Respiratory Distress 1}

The protein IGHMBP2, the loss of which causes SMARD1, was initially described as related to R-loop-dependent classswitch recombination [98]. Given the homologies in its sequence, IGHMBP2 is considered a member of the helicase superfamily 1 , and its biochemical characterization suggests ATP-dependent 5' $\rightarrow$ 3' DNA/RNA helicase activity [98]. The majority of the pathogenic mutations in SMARD1 patients are located in the helicase domain of IGHMBP2 [99, 100]. This information together with the similarity with the protein SETX suggests a probable link between IGHMBP2 defects and abnormal R-loop formation that should be investigated in the context of SMARD1.

\section{R-L oop Therapies}

R-loops are becoming increasingly interesting in the pathogenesis of neurodegenerative diseases and in the development of therapies to treat these conditions. If SMA and ALS share the pathogenic pathway of DNA damage induction driven by R-loops and consequent cellular death, then R-loop manipulation strategies would have importantimplications, including a possible therapeutic target role in multiple disorders. Therefore, it could be interesting to further investigate the Rloop-related pathogenesis of rare neurodegenerative disorders, such as SMARD1 and ALS4. The conspicuous number of alterations in RNA metabolism identified in different neuromuscular diseases raises the hypothesis of a common defect underling this spectrum of illnesses. This potentially common defect could provide possible therapeutic strategies not only for rare diseases but also other neurodegenerative disorders on this spectrum. R-loops also warrant investigation as therapeutic targets for diseases, such as SMA, that already have an available approved therapy. R-loop-based therapies could indeed provide additional benefits to SMA patients beyond the therapeutic effect of SMN restoration alone. However, despite their apparent importance in different pathogenic pathways, R-loops have yet to be fully exploited in drug design [57].

Numerous different compounds can bind to DNA/RNA hybrids, such as the aminoglycosides paramomycin and neomycin, the polyamides netropsin and distamycin, and ethidium bromide. These compounds intercalate into the hybrids and bind to the nucleic acid strands [101]. Despite their sensitivity to DNA/RNA hybrids, these compounds also recognize dsDNAs and RNAs, causing these agents to be mutagenic and potentially dangerous. This toxicity limits their application in clinical therapeutic strategies. However, recent studies demonstrated that a combination of these compounds, such as a combination of ethidium bromide derivatives and aminoglycosides, could bind more specifically to DNA/RNA hybrids, permitting the use of a subnanomolar dosage and providing a powerful tool for future R-loop-based therapy [101].

As an alternative, it is possible to influence R-loops by targeting some of the pathological mechanisms that favor their formation. For example, R-loops in trinucleotide repeat expansions could be modulated by targeting the expansions themselves [102]. Colak et al. (2014) demonstrated that a small molecule can inhibit R-loop generation at CGG repeats expansion in the FMR1 gene, preventing FMR1 silencingin 
fragile X syndrome (FXS) [103]. In C9ORF72 HRE pathology, resolving the quadruplex structures responsible for the stabilization of R-loops may represent a possible strategy to prevent the pathogenetic consequences of R-loops.

R-loop levels can also be modulated by targeting proteins physiologically involved in their metabolism. Upregulation of genes encoding R-loop-specific helicases, such as senataxin, demonstrated that DNA/RNA helicase activity is responsible for R-loop resolution at transcription termination sites [22, 32], potentially representing a promising strategy to treat pathology characterized by R-loop accumulation as noted in C9ORF72-associated diseases [61]. Similarly, modulation of ataxin could represent another therapeutic possibility. Ataxin was suggested as a possible R-loop regulator and is involved in numerous pathways related to R-loops, such as TDP-43 and FUS stress response pathway [67]. Overexpression of endonuclease RNase H1/H2 that specifically degrades RNA of RNA/DNA hybrids could represent another strategy to reduce R-loop levels and consequently ameliorate DNA damage, as observed in SH-SY5Y cells depleted of SMN [90]. Increases in the R-loop-resolving activity of these enzymes with specific ligands could be important for R-loop-related pathology. Finally, an alternative approach is to target the downstream effect of R-loops promoting DSB repair kinetics with chromatin-modulating drugs [61].

The possibility of targeting R-loops in different human diseases is exciting. It is important to remember that R-loops are ubiquitously distributed and are involved in different physiological processes to ensure that future treatments will be specific for pathologic mechanisms [57].

\section{Conclusion and Perspectives}

R-loops are becoming central molecular players in different neurologicaldisordersand are deserving ofincreasedattention in pathogenic theories and therapeutic strategies. R-loops form ubiquitously in different conditions and in different organisms and are necessary for main biological processes. However, R-loops can also be deleterious to cells, promoting DNA damage and genome instability. Distinguishing sbad Rloops $\wedge$ from Bgood R-loops $\wedge$ is fundamental in R-loop-based therapeutic research given that we do not know whether upregulation of R-loop modulators, such as senataxin, or administration of R-loop-resolving compounds could discriminate between these two forms.

There are two major issues in R-loop pathways: (1) How do distinct mutations in disease-related genes induce aberrant $\mathrm{R}$ loop formation? and (2) What are the consequences that lead to the different pathologies? In both cases, many pieces are missing; thus, we lack a full comprehension of R-looprelated diseases. However, some themes should be considered certainties. First, R-loop dysregulation is clearly connected with DNA damage, the consequences of which vary from cellular death to neurodegeneration. Second, R-loops have an active role in transcription regulation both locally and globally that contributes in particular to the pathogenesis of expansion diseases. Both of these pathological themes likely occur together in the same pathology, and other mechanisms are also likely involved.

Moreover, defining the roles of R-loops in motor neuron biology will be fundamental to understanding the selectivity of motorneuron deathinneurodegenerative disorders. Indeed, Sorrels et al. (2018) demonstrated that control of R-loop levels in neuronal cells during embryogenesis is crucial for the development of the central nervous system [95]. This information opens the possibility that R-loop-related motor neuron diseases have their pathogenic roots in early central nervous system development, which clearly could change many perspectives on these disorders.

In the future, studying R-loops in other human diseases will be relevant. This information will shed some light on R-loop pathways and could represent a step forward in the understanding of human diseases and our ability to treat them.

Acknowledgments Joint Programme Neurodegenerative Disease (JPND) Research grant DAMNDPATHS (2014) and ARISLA grant smallRNALS (2014) to SC and the Italian Ministry of Health RF2013-023555764 and Regione Lombardia TRANS-ALS to GPC are gratefully acknowledged. The authors wish to thank the Associazione Centro Dino Ferrari for its support.

\section{References}

1. Taylor JP, Hardy J, Fischbeck KH (2002) Toxic proteins in neurodegenerative disease. Science 296(80):1991-1995. https://doi. org/10.1126/science.1067122

2. Takalo M, Salminen A, Soininen H et al (2013) Protein aggregation and degradation mechanisms in neurodegenerative diseases. Am J Neurodegener Dis 2:1-14

3. Barker HV, Niblock M, Lee Y-B et al (2017) RNA misprocessing in C9orf72-linked neurodegeneration. Front Cell Neurosci 11. https://doi.org/10.3389/fncel.2017.00195

4. Paulsen RD, Soni DV, Wollman R et al (2009) A genome-wide siRNA screen reveals diverse cellularprocesses and pathways that mediate genome stability. Mol Cell 35:228-239. https://doi.org/ 10.1016/j.molcel.2009.06.021

5. Li DK, Tisdale S, Lotti F, Pellizzoni L (2014) SMN control of RNP assembly: frompost-transcriptional generegulation tomotor neuron disease. Semin Cell Dev Biol 32:22-29

6. Yanling Zhao D, Gish G, Braunschweig U et al (2016) SMN and symmetric arginine dimethylation of RNA polymerase II Cterminal domain control termination. Nature 529:48-53. https:// doi.org/10.1038/nature16469

7. Chédin F (2016) Nascent connections: R-loops and chromatin patterning. Trends Genet 32:828-838

8. Roy D, Zhang Z, Lu Z et al (2010) Competition between the RNA transcript and the nontemplate DNA strand during R-loop formation in vitro: a nick can serve as a strong R-loop initiation site. Mol Cell Biol 30:146-159. https://doi.org/10.1128/MCB.00897-09 
9. Roberts R, Crothers D (1992) Stability and properties of double and triple helices: Dramatic effects of RNA or DNA backbone composition. Science 258(80):1463-1466. https://doi.org/10. 1126/science.1279808

10. WestoverKD, Bushnell DA, Kornberg RD (2004) Structural basis of transcription: separation of RNA from DNA by RNA polymerase II. Science 303:1014-1016. https://doi.org/10.1126/science. 1090839

11. Elden AC, Kim HJ, Hart MP et al (2010) Ataxin-2 intermediatelength polyglutamine expansions are associated with increased risk for ALS. Nature 466:1069-1075. https://doi.org/10.1038/ nature09320

12. Santos-Pereira JM, Aguilera A(2015) Rloops: new modulators of genome dynamics and function. Nat Rev Genet 16:583-597

13. Aguilera A, García-Muse T (2012) R loops: from transcription byproducts to threats to genome stability. Mol Cell 46:115-124

14. Skourti-Stathaki K, Proudfoot NJ (2014) A double-edged sword: $\mathrm{R}$ loops as threats to genome integrity and powerful regulators of gene expression. Genes Dev 28:1384-1396

15. Duquette ML, Handa P, Vincent JA et al (2004) Intracellular transcription of G-rich DNAs induces formation of G-loops, novel structures containing G4 DNA. Genes Dev 18:1618-1629. https://doi.org/10.1101/gad.1200804

16. Skourti-Stathaki K, Proudfoot NJ, Gromak N (2011) Human senataxin resolves RNA/DNA hybrids formed at transcriptional pause sites to promote Xrn2-dependent termination. Mol Cell 42: 794-805. https://doi.org/10.1016/j.molcel.2011.04.026

17. Denis MM, Tolley ND, Bunting $M$ et al (2005) Escaping the nuclear confines: signal-dependent pre-mRNA splicing in anucleate platelets. Cell 122:379-391. https://doi.org/10.1016/j. cell.2005.06.015

18. Domínguez-Sánchez MS, Barroso S, Gómez-González B et al (2011) Genome instability and transcription elongation impairment in human cells depleted of THO/TREX. PLoS Genet 7: e1002386. https://doi.org/10.1371/journal.pgen.1002386

19. D'Alessandro G, d'Adda di Fagagna F (2017) Transcription and DNA damage: holding hands or crossing swords? J Mol Biol 429: 3215-3229

20. Richard P,Manley JL (2017) R loops and links to human disease. J Mol Biol 429:3168-3180

21. Grabczyk E, Mancuso M, Sammarco MC (2007) A persistent RNA. DNA hybridformed bytranscription of theFriedreichataxia triplet repeat in live bacteria, and by T7 RNAP in vitro. Nucleic Acids Res 35:5351-5359. https://doi.org/10.1093/nar/gkm589

22. Chen Y-Z, Bennett CL, Huynh HM et al (2004) DNA/RNA helicase gene mutations in a form of juvenile amyotrophic lateral sclerosis (ALS4). Am J Hum Genet 74:1128-1135. https://doi. org/10.1086/421054

23. Steinmetz EJ, Warren CL, Kuehner JN, Panbehi B, Ansari AZ, Brow DA (2006) Genome-wide distribution of yeast RNA polymerase II and its control by Sen1 helicase. Mol Cell 24(5):735746. https://doi.org/10.1016/j.molcel.2006.10.023

24. Lagier-Tourenne C, Polymenidou M, Hutt KR et al (2012) Divergent roles of ALS-linked proteins FUS/TLS and TDP-43 intersect in processing long pre-mRNAs. Nat Neurosci 15:14881497. https://doi.org/10.1038/nn.3230

25. Nonhoff U, Ralser M, Welzel F et al (2007) Ataxin-2 interacts with the DEAD/H-box RNA helicase DDX6 and interferes with P-bodiesandstress granules. MolBiolCell 18:1385-1396.https:// doi.org/10.1091/mbc.E06-12-1120

26. Moreira MC, Klur S, Watanabe M et al (2004) Senataxin, the ortholog of a yeast RNA helicase, is mutant in ataxia-ocular apraxia 2. Nat Genet 36:225-227. https://doi.org/10.1038/ng1303

27. Bassuk AG, Chen YZ, Batish SD et al (2007) In cis autosomal dominant mutation of senataxin associated with tremor/ataxia syndrome. Neurogenetics 8:45-49. https://doi.org/10.1007/ s10048-006-0067-8

28. Balk B, Maicher A, Dees M, Klermund J, Luke-Glaser S, Bender K, Luke B (2013) Telomeric RNA-DNA hybrids affect telomerelength dynamics and senescence. Nat Struct Mol Biol 20(10): 1199-1205. https://doi.org/10.1038/nsmb.2662

29. De Amicis A, Piane M, Ferrari F et al (2011) Role of senataxin in DNA damage and telomeric stability. DNA Repair (Amst) 10: 199-209. https://doi.org/10.1016/j.dnarep.2010.10.012

30. Sun S, Ling SC, Qiu J et al (2015) ALS-causative mutations in FUS/TLS confer gain and loss of function by altered association with SMN and U1-snRNP. Nat Commun 6:6171. https://doi.org/ 10.1038/ncomms7171

31. Hua Y, Zhou J (2004) Survival motor neuron protein facilitates assembly of stress granules. FEBS Lett 572:69-74. https://doi.org/ 10.1016/j.febslet.2004.07.010

32. Groh M, Albulescu LO, Cristini A, Gromak N (2017) Senataxin: genome guardian at the interface of transcription and neurodegeneration. J Mol Biol 429:3181-3195

33. Freudenreich CH (2018) R-loops: targets for nuclease cleavage and repeat instability. Curr Genet:1-6

34. Lin Y,Dent SYR, Wilson JH et al (2010) R loops stimulate genetic instability of CTG · CAG repeats. PNAS 107:692-697.https://doi. org/10.1073/pnas.0909740107

35. Reddy K, Schmidt MHM, Geist JM et al (2014) Processing of do uble-R-loops in ( CAG) •(CTG) and C9orf 72 (GGGGCC)•(GGCCCC) repeats causes instability. Nucleic Acids Res 42:10473-10487.https://doi.org/10.1093/nar/gku658

36. Zhao XN, Usdin K (2015) The repeat expansion diseases: The dark side of DNA repair. DNA Repair (Amst) 32:96-105. https://doi.org/10.1016/j.dnarep.2015.04.019

37. Hardiman O, Al-Chalabi A, Chio A, Corr EM, Logroscino G, Robberecht W, Shaw PJ, Simmons Z, van den Berg LH (2017) Amyotrophic lateral sclerosis. Nat Rev Dis Primers 3:17085. https://doi.org/10.1038/nrdp.2017

38. Bucchia M, Ramirez A, Parente Vet al (2015) Therapeutic development in amyotrophic lateral sclerosis. Clin Ther 37:668-680

39. Al-Chalabi A, Van Den Berg LH, Veldink J (2017) Gene discovery in amyotrophic lateral sclerosis: implications for clinical management. Nat Rev Neurol 13:96-104

40. Salvi JS, Mekhail K (2015) R-loops highlight the nucleus in ALS. Nucleus 6:23-29. https://doi.org/10.1080/19491034.2015. 1004952

41. DeJesus-Hernandez M, Mackenzie IR, Boeve BF et al (2011) Expanded GGGGCC hexanucleotide repeat in noncoding region of C9ORF72 causes chromosome 9p-linked FTD and ALS. Neuron 72:245-256. https://doi.org/10.1016/j.neuron.2011.09. 011

42. Renton AE, Majounie E, Waite A et al (2011) A hexanucleotide repeat expansion in C9ORF72 is the cause of chromosome 9p21linked ALS-FTD. Neuron 72:257-268. https://doi.org/10.1016/j. neuron.2011.09.010

43. Majounie E, Renton AE, Mok K et al (2012) Frequency of the C9orf72 hexanucleotide repeat expansion in patients with amyotrophic lateral sclerosis and frontotemporal dementia: a crosssectional study. Lancet Neurol 11:323-330. https://doi.org/10. 1016/S1474-4422(12)70043-1

44. Rutherford NJ, Heckman MG, DeJesus-Hernandez M et al (2012) Length of normal alleles of C9ORF72 GGGGCC repeat do not influence disease phenotype. Neurobiol Aging 33:2950.e52950.e7. https://doi.org/10.1016/j.neurobiolaging.2012.07.005

45. Haeusler AR, Donnelly CJ, Periz G et al (2014) C9orf72 nucleotiderepeat structures initiatemolecularcascades of disease. Nature 507:195-200. https://doi.org/10.1038/nature13124

46. Fratta P, Mizielinska S, Nicoll AJ et al (2012) C9orf72 hexanucleotide repeat associated with amyotrophic lateral 
sclerosis and frontotemporal dementia forms RNA Gquadruplexes. Sci Rep 2. https://doi.org/10.1038/srep01016

47. Ciura S, Lattante S, Le Ber I et al (2013) Loss of function of C9orf72 causes motor deficits in a zebrafish model of amyotrophic lateral sclerosis. Ann Neurol 74:180-187. https://doi.org/10. 1002/ana.23946

48. Waite AJ, Bäumer D, East S et al (2014) Reduced C9orf72 protein levels in frontal cortex of amyotrophic lateral sclerosis and frontotemporal degeneration brain with the C9ORF72 hexanucleotide repeat expansion. Neurobiol Aging 35:1779.e51779.e13. https://doi.org/10.1016/j.neurobiolaging.2014.01.016

49. Reddy K, Tam M, Bowater RP et al (2011) Determinants of Rloop formation at convergent bidirectionally transcribed trinucleotide repeats. Nucleic Acids Res 39:1749-1762. https://doi.org/ 10.1093/nar/gkq935

50. Hensman DJ, Poulter M, Beck J et al (2014) C9orf72 expansions are the most common genetic cause of Huntington disease phenocopies. Neurology 82:292-299. https://doi.org/10.1212/ WNL.0000000000000061

51. Belzil VV, Bauer PO, Prudencio M et al (2013) Reduced C9orf72 gene expression in c9FTD/ALS is caused by histone trimethylation, an epigenetic event detectable in blood. Acta Neuropathol 126:895-905. https://doi.org/10.1007/s00401-0131199-1

52. Xi Z, Zinman L, Moreno D et al (2013) Hypermethylation of the CpG island near the G4C2 repeat in ALS with a C9orf72 expansion. Am J Hum Genet 92:981-989. https://doi.org/10.1016/j. ajhg.2013.04.017

53. Liu EY, Russ J, Wu K et al (2014) C9orf72 hypermethylation protects against repeat expansion-associated pathology in ALS/ FTD. Acta Neuropathol 128:525-541. https://doi.org/10.1007/ s00401-014-1286-y

54. Russ J, Liu EY, Wu K et al (2015) Hypermethylation of repeat expanded C9orf72 is a clinical and molecular disease modifier. Acta Neuropathol 129:39-52. https://doi.org/10.1007/s00401014-1365-0

55. Belzil VV, Bauer PO, Gendron TF et al (2014) Characterization of DNA hypermethylation in the cerebellum of c9FTD/ALS patients. Brain Res 1584:15-21. https://doi.org/10.1016/j. brainres.2014.02.015

56. Xi Z, Rainero I, Rubino E et al (2014) Hypermethylation of the CpG-island near the C9orf72 G4C2-repeat expansion in FTLD patients. Hum Mol Genet 23:5630-5637. https://doi.org/10. 1093/hmg/ddu279

57. Groh M, Gromak N (2014) Out of balance: R-loops in human disease. PLoS Genet 10:e1004630

58. Ginno PA, Lott PL, Christensen HC et al (2012) R-loop formation is a distinctive characteristic of unmethylated human CpG Island promoters. Mol Cell 45:814-825. https://doi.org/10.1016/j. molcel.2012.01.017

59. Wang J, Haeusler AR, Simko EA (2015) Emerging role of rna-dna hybrids in c9orf72-linked neurodegeneration. Cell Cycle 14:526-532

60. Esanov R, Cabrera GT, Andrade NS et al (2017) A C9ORF72 BAC mouse model recapitulates key epigeneticperturbations of ALS/FTD. Mol Neurodegener 12:46. https://doi.org/10.1186/ s13024-017-0185-9

61. Walker C, Herranz-Martin S, Karyka E et al (2017) C9orf72 expansion disrupts ATM-mediated chromosomal break repair. Nat Neurosci 20:1225-1235. https://doi.org/10.1038/nn.4604

62. van Blitterswijk M, Mullen B, Heckman MG et al(2014) Ataxin-2 as potential disease modifier in C9ORF72 expansion carriers. Neurobiol Aging 35:2421.e13-2421.e17. https://doi.org/10. 1016/j.neurobiolaging.2014.04.016

63. Pulst S-M, Nechiporuk A, Nechiporuk T et al (1996) Moderate expansion of a normally biallelic trinucleotide repeat in spinocerebellar ataxia type 2. Nat Genet 14:269-276. https://doi. org/10.1038/ng1196-269

64. Sanpei K, Takano H, Igarashi S et al (1996) Identification of the spinocerebellar ataxia type 2 gene using a direct identification of repeat expansion and cloning technique, DIRECT. Nat Genet 14: 277-284. https://doi.org/10.1038/ng1196-277

65. Farg MA, Soo KY, Warraich ST et al (2013) Ataxin-2 interacts with FUS and intermediate-length polyglutamine expansions enhance FUS-related pathology in amyotrophic lateral sclerosis. Hum Mol Genet 22:717-728. https://doi.org/10.1093/hmg/ dds479

66. Salvi JS, Chan JNY, Szafranski K et al (2014) Roles for Pbp1 and caloric restriction in genome and lifespan maintenance via suppression of RNA-DNAhybrids. DevCell30:177-191. https://doi. org/10.1016/j.devcel.2014.05.013

67. Becker LA, Huang B, Bieri G et al (2017) Therapeutic reduction of ataxin-2 extends lifespan and reduces pathology in TDP-43 mice. Nature 544:367-371. https://doi.org/10.1038/nature22038

68. Neumann M, Sampathu DM, Kwong LK et al (2006) Ubiquitinated TDP-43 in frontotemporal lobar degeneration and amyotrophic lateral sclerosis. Science 314(80):130-133. https:// doi.org/10.1126/science.1134108

69. Colombrita C, Zennaro E, Fallini C et al (2009) TDP-43 is recruited to stress granules in conditions of oxidative insult. J Neurochem 111: 1051-1061. https://doi.org/10.1111/j.1471-4159.2009.06383.x

70. Kwiatkowski TJ, Bosco DA, Leclerc AL et al (2009) Mutations in theFUS/TLS gene on chromosome 16 cause familial amyotrophic lateral sclerosis. Science 323:1205-1208.https://doi.org/10.1126/ science.1166066

71. Alami NH, Smith RB, Carrasco MA et al (2014) Axonal transport of TDP-43 mRNA granules is impaired by ALS-causing mutations. Neuron 81:536-543. https://doi.org/10.1016/j.neuron. 2013.12.018

72. Mancini C, Orsi L, Guo Y et al (2015) An atypical form of AOA2 with myoclonus associated with mutations in SETX and AFG3L2. BMC Med Genet 16:16. https://doi.org/10.1186/s12881-015-0159-0

73. Steinmetz EJ, Conrad NK, Brow DA, Corden JL (2001) RNAbinding protein Nrd1 directs poly(A)-independent 3???-end formation of RNA polymerase II transcripts. Nature 413:327-331. https://doi.org/10.1038/35095090

74. Mischo HE, Gómez-González B, Grzechnik P et al (2011)Yeast Sen1 helicase protects the genome from transcription-associated instability. Mol Cell 41:21-32. https://doi.org/10.1016/j.molcel. 2010.12.007

75. Hatchi E, Skourti-Stathaki K, Ventz S et al (2015) BRCA1 recruitment to transcriptional pause sites is required for R-loop-driven DNA damage repair. Mol Cell 57:636-647. https://doi.org/10. 1016/j.molcel.2015.01.011

76. Yuce O, West SC (2013) Senataxin, defective in the neurodegenerative disorder ataxia with oculomotor apraxia 2, lies at the interface of transcription and the DNA damageresponse. Mol Cell Biol 33:406-417. https://doi.org/10.1128/MCB.01195-12

77. Richard P, Feng S, Manley JL (2013) A SUMO-dependent interaction between senataxin and the exosome, disrupted in the neurodegenerative disease AOA2, targets the exosome to sites of transcription-induced DNA damage. Genes Dev 27:2227-2232. https://doi.org/10.1101/gad.224923.113

78. Richard P, Manley JL (2014) SETX sumoylation: a link between DNA damage and RNA surveillance disrupted in AOA2. Rare Dis (Austin, Tex) 2:e27744. https://doi.org/10.4161/rdis.27744

79. Becherel OJ, Yeo AJ, Stellati A et al (2013) Senataxin plays an essential role with DNA damage response proteins in meiotic recombination and gene silencing. PLoS Genet 9:e1003435. https:// doi.org/10.1371/journal.pgen.1003435

80. Yeo AJAAJ, Becherel OJO, Luff JE et al (2014) R-loops in proliferating cells but not in the brain: implications for AOA2 and 
other autosomal recessive ataxias. PLoS One 9:e90219. https:// doi.org/10.1371/journal.pone.0090219

81. Graf M, Bonetti D, Lockhart A et al (2017) Telomere length determines TERRA and R-loop regulation through the cell cycle. Cell 170:72-85.e14. https://doi.org/10.1016/j.cell.2017.06.006

82. Grunseich C, Wang IX, Watts JA et al (2018) Senataxin mutation reveals how R-loops promote transcription by blocking DNA methylation at gene promoters. Mol Cell 69:426-437.e7. https:// doi.org/10.1016/j.molcel.2017.12.030

83. Cohen S, Puget N, Lin YL et al (2018) Senataxin resolves RNA: DNA hybrids forming at DNA double-strand breaks to prevent translocations. Nat Commun 9:533. https://doi.org/10.1038/ s41467-018-02894-w

84. Hill SJ, Rolland T, Adelmant G et al (2014) Systematic screening reveals a role for BRCA1 in the response to transcriptionassociated DNA damage. Genes Dev 28:1957-1975. https://doi. org/10.1101/gad.241620.114

85. Lagier-Tourenne C, Cleveland DW (2009) Rethinking ALS: the FUS about TDP-43. Cell 136:1001-1004

86. Ling SC, Polymenidou M, Cleveland DW (2013) Converging mechanisms in als and FTD: disrupted RNA and protein homeostasis. Neuron 79:416-438

87. Han H, Irimia M, Ross PJ et al (2013) MBNL proteins repress EScell-specific alternative splicing and reprogramming. Nature 498: 241-245. https://doi.org/10.1038/nature12270

88. Ayala YM, De Conti L, Avendaño-Vázquez SE et al (2011) TDP43 regulates its mRNA levels through a negative feedback loop. EMBO J 30:277-288. https://doi.org/10.1038/emboj.2010.310

89. Hill SJ, Mordes DA, Cameron LA et al (2016) Two familial ALS proteins function in prevention/repair of transcription-associated DNA damage. Proc Natl Acad Sci 113:E7701-E7709. https://doi. org/10.1073/pnas.1611673113

90. Jangi M, Fleet C, Cullen P et al (2017) SMN deficiency in severe models of spinal muscular atrophy causes widespread intron retention and DNA damage. Proc Natl Acad Sci 114:E2347-E2356. https://doi.org/10.1073/pnas.1613181114

91. Arnold WD, Kassar D, Kissel JT (2015) Spinal muscular atrophy: diagnosis and management in a new therapeutic era. Muscle Nerve 51:157-167. https://doi.org/10.1002/mus.24497
92. Parente V, Corti S (2018) Advances in spinal muscular atrophy therapeutics. Ther Adv Neurol Disord 11:175628561875450. https://doi.org/10.1177/1756285618754501

93. Porro F, Rinchetti P, Magri F et al (2014) The wide spectrum of clinical phenotypes of spinal muscular atrophy with respiratory distress type 1: a systematic review. J Neurol Sci 346:35-42

94. Vanoli F, Rinchetti P, Porro F et al (2015) Clinical and molecular features and therapeutic perspectives of spinal muscular atrophy with respiratory distress type 1. J Cell Mol Med 19:2058-2066. https://doi.org/10.1111/jcmm.12606

95. Sorrells S, Nik S, Casey M et al (2018) Spliceosomal components protect embryonic neurons from R-loop-mediated DNA damage and apoptosis. Dis Model Mech 11:dmm.031583. https://doi.org/ 10.1242/dmm.031583

96. Wahl MC, Will CL, Lührmann R (2009) The spliceosome: design principles of a dynamic RNP machine. Cell 136:701-718

97. Burghes AHM, Beattie CE (2009) Spinal muscular atrophy: why do low levels of survival motor neuron protein make motor neurons sick? Nat Rev Neurosci 10:597-609

98. Fukita Y,Mizuta TR, Shirozu Met al (1993) The human S $\mu b p-2$, a DNA-binding protein specific to the single-stranded guanine-rich sequence related to the immunoglobulin $\mu$ chain switch region. $J$ Biol Chem 268:17463-17470

99. Guenther UP, Handoko L, Laggerbauer B et al (2009) IGHMBP2 is a ribosome-associated helicase inactive in the neuromuscular disorder distal SMA type 1 (DSMA1). Hum Mol Genet 18: 1288-1300. https://doi.org/10.1093/hmg/ddp028

100. Lim SC, Bowler MW, Lai TF, Song H (2012) The Ighmbp2 helicase structure reveals the molecular basis for disease-causing mutations in DMSA1. Nucleic Acids Res 40:11009-11022. https://doi.org/10.1093/nar/gks792

101. Shaw NN, Xi H, Arya DP (2008) Molecular recognition of a DNA:RNA hybrid: sub-nanomolar binding by a neomycinmethidium conjugate. Bioorganic Med Chem Lett 18:41424145. https://doi.org/10.1016/j.bmcl.2008.05.090

102. McIvor EI, Polak U, Napierala M (2010) New insights into repeat instability: role of RNA•DNA hybrids. RNA Biol 7:551-558

103. Colak D, Zaninovic N, Cohen MS et al (2014) Promoter-bound trinucleotiderepeatmRNA drives epigenetic silencing in fragile $\mathrm{X}$ syndrome. Science 343(80):1002-1005. https://doi.org/10.1126/ science.1245831 